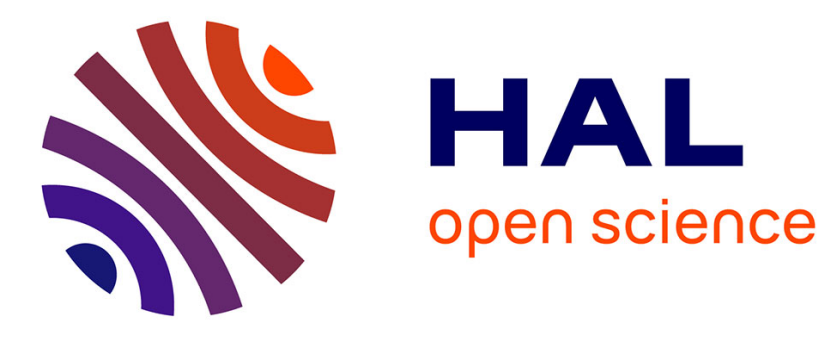

\title{
The ORP on-sky community access program for adaptive optics instrumentation development
}

Tim Morris, James Osborn, Marcos Reyes, Iciar Montilla, Gérard Rousset, Éric Gendron, Thierry Fusco, Benoit Neichel, Simone Esposito, Paulo Garcia, et al.

\section{To cite this version:}

Tim Morris, James Osborn, Marcos Reyes, Iciar Montilla, Gérard Rousset, et al.. The ORP onsky community access program for adaptive optics instrumentation development. Adaptive Optics Systems VII, Dec 2020, Online Only, United States. pp.181, 10.1117/12.2562805 • hal-03224500

\section{HAL Id: hal-03224500 https://hal.science/hal-03224500}

Submitted on 9 Jun 2021

HAL is a multi-disciplinary open access archive for the deposit and dissemination of scientific research documents, whether they are published or not. The documents may come from teaching and research institutions in France or abroad, or from public or private research centers.
L'archive ouverte pluridisciplinaire $\mathbf{H A L}$, est destinée au dépôt et à la diffusion de documents scientifiques de niveau recherche, publiés ou non, émanant des établissements d'enseignement et de recherche français ou étrangers, des laboratoires publics ou privés. 


\title{
The ORP on-sky community access program for adaptive optics instrumentation development
}

\author{
T. Morris ${ }^{\mathrm{a}}$, J. Osborn ${ }^{\mathrm{a}}$, M. Reyes ${ }^{\mathrm{b}}$, I. Montilla ${ }^{\mathrm{b}}$, G. Rousset ${ }^{\mathrm{c}}$, E. Gendron ${ }^{\mathrm{c}}$, T. Fusco ${ }^{\mathrm{d}, \mathrm{e}}$, B. \\ Neichel $^{\mathrm{e}}$, S. Esposito ${ }^{\mathrm{f}}$, P.J.V Garcia ${ }^{\mathrm{g}}$, C. Kulcsar ${ }^{\mathrm{h}}$, C. Correia ${ }^{\mathrm{g}}$, J-L Beuzite ${ }^{\mathrm{e}}$, N.A. Bharmal ${ }^{\mathrm{a}}$, L. \\ Bardou $^{\mathrm{a}}$, L. Staykov ${ }^{\mathrm{a}}$, and D. Bonaccini Calia ${ }^{\mathrm{i}}$ \\ ${ }^{a}$ Durham University, Department of Physics, South Road, Durham, UK

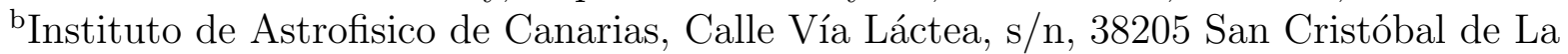 \\ Laguna, Santa Cruz de Tenerife, Spain \\ ${ }^{\mathrm{c} O b s e r v a t o i r e ~ d e ~ P a r i s ~-~ L E S I A, ~ O b s e r v a t o i r e ~ d e ~ P a r i s, ~ S e c t i o n ~ d e ~ M e u d o n, ~} 5$ place Jules \\ Janssen, 92195 Meudon, France \\ ${ }^{\mathrm{d} O N E R A,} 29$ Avenue de la Division Leclerc, 92320 Châtillon, France

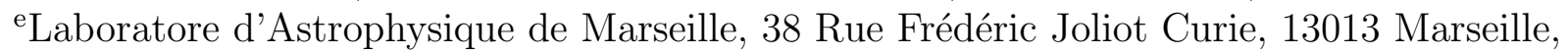 \\ France

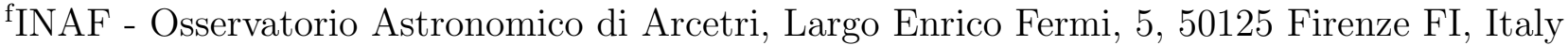 \\ ${ }^{g}$ University of Porto, Departamento de Engenharia Física, Rua Dr. Roberto Frias, 4200-465 \\ Porto, Portugal \\ ${ }^{\mathrm{h}}$ L'Institut d'Optique Graduate School, 2 Avenue Augustin Fresnel, 91120 Palaiseau, France \\ ${ }^{i}$ European Southern Observatory, Karl-Schwarzschild-Strasse 2, 85748 Garching bei München, \\ Germany
}

\begin{abstract}
On-sky testing of new instrumentation concepts is required before they can be incorporated within facility-class instrumentation with certainty that they will work as expected within a real telescope environment. Increasingly, many of these concepts are not designed to work in seeing-limited conditions and require an upstream adaptive optics system for testing. Access to on-sky AO systems to test such systems is currently limited to a few research groups and observatories worldwide, leaving many concepts unable to be tested. A pilot program funded through the H2020 OPTICON program offering up to 15 nights of on-sky time at the CANARY Adaptive Optics demonstrator is currently running but this ends in 2021. Pre-run and on-sky support is provided to visitor experiments by the CANARY team. We have supported 6 experiments over this period, and plan one more run in early 2021. We have recently been awarded for funding through the H2020 OPTICON-RADIO PILOT call to continue and extend this program up until 2024, offering access to CANARY at the $4.2 \mathrm{~m}$ William Herschel Telescope and 3 additional instruments and telescopes suitable for instrumentation development. Time on these facilities will be open to researchers from across the European research community and time will be awarded by answering a call for proposals that will be assessed by an independent panel of instrumentation experts. Unlike standard observing proposals we plan to award time up to 2 years in advance to allow time for the visitor instrument to be delivered. We hope to announce the first call in mid-2021. Here we describe the facilities offered, the support available for on-sky testing and detail the eligibility and application process.
\end{abstract}

Keywords: Adaptive optics, On-sky testing, instrument development

\section{INTRODUCTION}

On-sky testing of new adaptive optics instrumentation is a key step in the adoption of new techniques and technologies within facility-class astronomical instrumentation. Instrumentation development using existing

Further author information: T.J.M.: E-mail: t.j.morris@durham.ac.uk 
European AO facilities is not possible because these systems typically cannot be modified without impacting their astronomical science programs, severely limiting access. Testing of new post-focal instrumentation techniques and concepts is best undertaken at dedicated on-sky test facilities, however these facilities are costly to build and operate. Access to on-sky test facilities is typically limited to research groups and institutes that have been directly involved in the development of a given $\mathrm{AO}$ system, which limits the ability of the wider community to develop new instrumentation concepts that rely on an AO-corrected focus.

Through the H2020's OPTICON (2016-2020) project we have developed and operated an access program ${ }^{1}$ that allows research groups across the European research community access to telescopes for the specific task of testing new instrumentation and AO concepts. This program funded visitor access to the CANARY Adaptive Optics (AO) system explicitly for the purpose of technology development. In addition to a set of on-sky runs held in 2019 supporting astronomical AO experiments from 27 instrumentation researchers from 5 countries, we have also been able to provide access to users outside astronomy for the development of optical communications technologies. Experiments supported in 2019-2021 under the OPTICON program include:

- Tests of control system performance optimisation using data-driven models to control low-order wavefront modes $^{2}$

- Diffraction-limited multi-core IFU with an astrophotonic-fed high resolution spectrograph ${ }^{3}$

- A discrete beam combiner for pupil interferometry ${ }^{4}$

- A post-focal high-efficiency point diffraction interferometer for high-contrast $\mathrm{AO}^{5}$

- A Mach-Zehnder based wavefront sensor for high-contrast observations ${ }^{6}$

- An adiabatic-tapered fibre feed and high-resolution single-mode spectrograph

- Uplink tip-tilt correction for sodium LGS using auxiliary telescopes

- A new low-cost turbulence scintillation-based profiling instrument

This access program has recently been extended for 4 years through the OPTICON-RADIONET Pilot (ORP) project. In this paper we describe the facilities on offer to the community in Section 2, the support and funding available to researchers that are awarded on-sky time in section 3 and how time will be awarded in section 4 .

\section{FACILITIES OFFERED}

We offer access to several instruments installed across the Observatorio del Roque de los Muchachos (ORM) in La Palma, Spain. These instruments were all originally developed for the CANARY project, ${ }^{7}$ but now comprise the core of the test facility on offer through this program. Each instrument can be operated independently of the other systems and proposals can make use of any or all of the facilities on offer. We expect that the majority of proposals will require only the use of existing CANARY infrastructure, the installation of an experimental component at the AO-corrected focus, or modifications to the real-time control system for testing e.g. new controllers or interfacing new cameras. You are welcome to request the use of any or all parts of the CANARY system, ranging from the full tomographic AO system with sodium LGS, to SCAO on bright objects, or observations that require the INT only. The location of the facilities with respect to one another is shown in Figure 1.

In the following subsections we provide more details of the three facilities on offer through this program; CANARY installed on the $4.2 \mathrm{~m}$ William Herschel Telescope, the 'Wendelstein' Laser Guide Star Unit, and two instruments that can be installed on the $2.5 \mathrm{~m}$ Isaac Newton Telescope. 


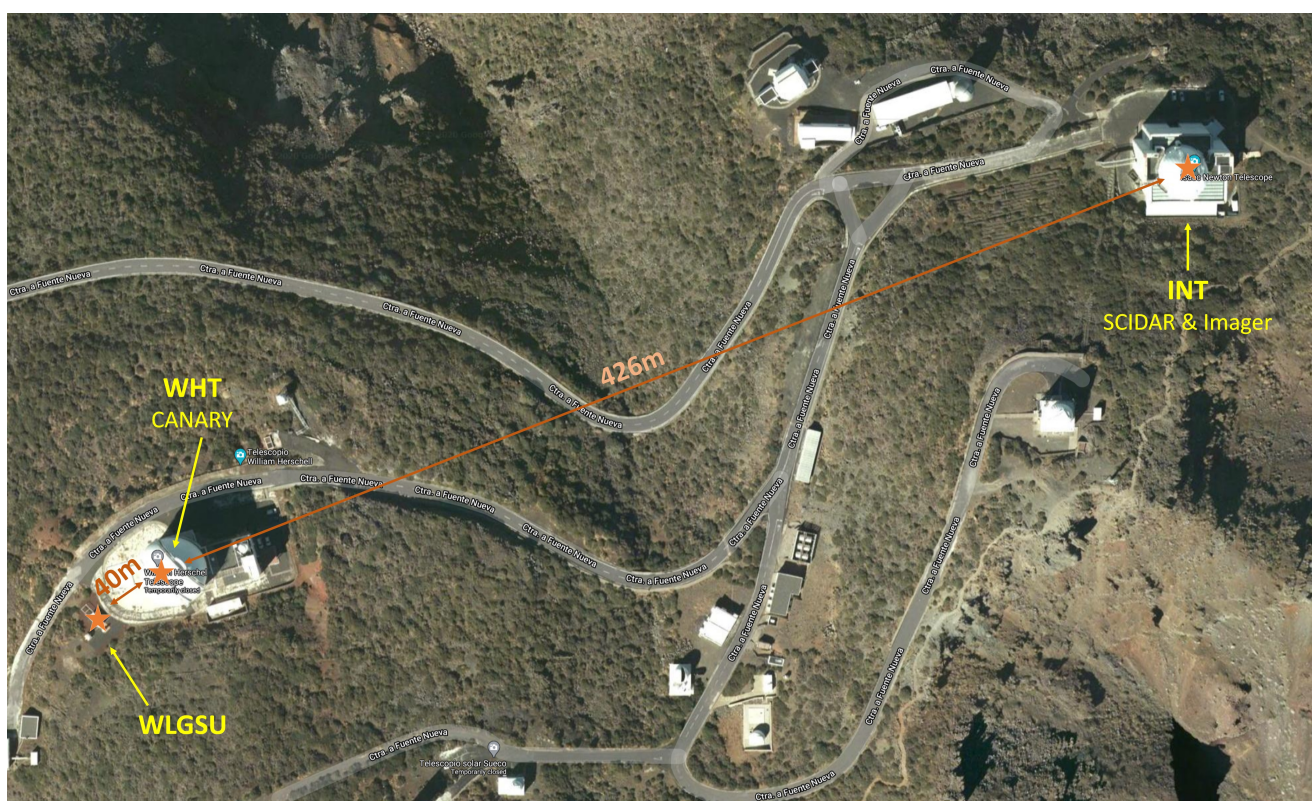

Figure 1. Plan view of observatory site showing locations of telescopes that host facilities offered through the ORP on-sky access program. Image (C)Google

\subsection{CANARY}

CANARY is an AO demonstrator installed at the $4.2 \mathrm{~m}$ William Herschel Telescope (La Palma, Spain) that was developed by a large international collaboration led by Durham University and LESIA between 2009-2017 to demonstrate wide-field and laser guide star AO techniques in preparation for the next generation Extremely Large Telescope. CANARY is the only wide-field AO test bench worldwide that is dedicated to AO research and development and is installed on a gravity-stable Nasmyth platform with a large free area available for visitor instrumentation. CANARY has provided the first on-sky demonstrations of many of the wide-field AO operating modes that almost all future telescope AO systems rely on, and remains the only AO system on a 4 m-class telescope that is dedicated to AO development. The CANARY configuration offered for observations under this program supports a variety of AO operating modes including SCAO and multi-NGS tomographic AO. The system comprises the following :

- Closed-loop tomographic NGS mode, with 3 off-axis NGS Shack-Hartmann wavefront sensors that can be positioned within a 2.5 arcminute derotated field

- An on-axis NGS Shack-Hartmann wavefront sensor for calibration and SCAO operation

- An on-axis sodium LGS Shack-Hartmann wavefront sensor on a removable kinematic bench

- A low-order 52-actuator closed-loop DM and tip/tilt mirror

- A high-order 241-actuator DM that can be operated in closed-loop SCAO or open-loop tomographic modes.

- Approximately 1 x $1.5 \mathrm{~m}$ of free bench space for visitor instruments fed with a 1 arcminute diameter $\mathrm{f} / 11$ $\mathrm{AO}$ corrected beam.

- A reconfigurable real-time control system running on standard CPU machines, written in C.

- A comprehensive telescope simulator containing NGS and LGS sources, as well as two turbulent phase screens and a range of pupil alignment and calibration masks 
CANARY can host a variety of optical instruments at its AO corrected output and also test new AO calibration and control schemes through modification of the real-time control system. Modifications to the main CANARY system are also supported and we have a replaceable mezzanine optical bench where new wavefront sensing systems can be installed and integrated within the main AO control loop. The CANARY optical bench and approximate space envelope available for visitor instrumentation is shown in Figure 2.
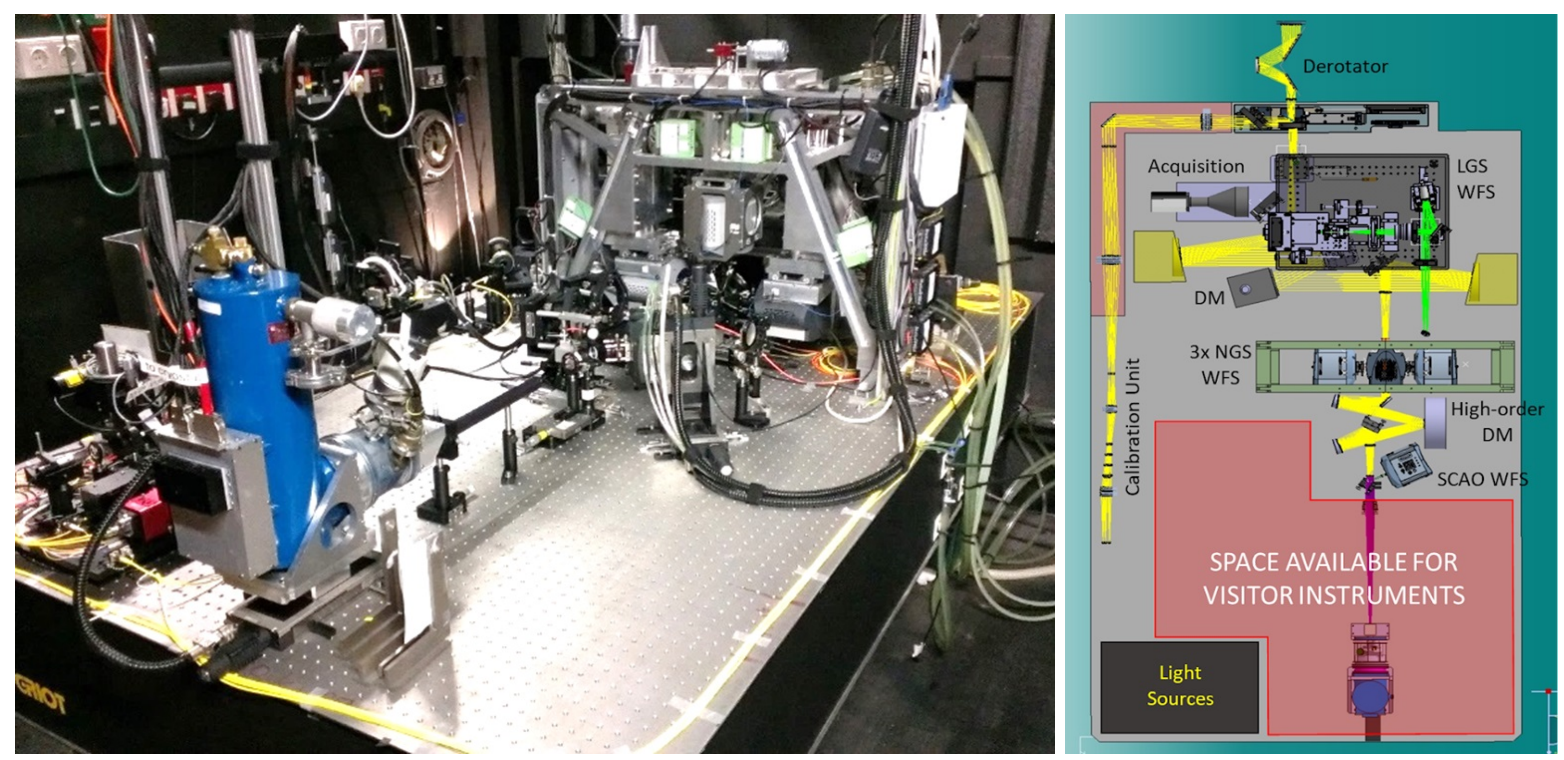

Figure 2. CANARY optical bench installed in the Nasmyth platform of the WHT (left) and schematic overview of CANARY bench showing space available for visitor instrumentation (right)

\subsection{Isaac Newton Telescope}

We also have two AO monitoring instruments that can be installed on the $2.5 \mathrm{~m}$ Isaac Newton Telescope (INT). The first of these is a Stereo-SCIDAR ${ }^{8}$ instrument that can provide a high vertical resolution turbulence and wind velocity profiles. The Stereo-SCIDAR has a vertical resolution in the region of 100-300 $\mathrm{m}$ and can profile up to $15-20 \mathrm{~km}$ dependent on the asterism being observed. Profiles can be provided every 2 minutes during operation.

We also offer a wide-field 6.6 arcminute diameter $589 \mathrm{~nm}$ imager that has a pixel scale of approximately 0.15 arcseconds. The INT is $420 \mathrm{~m}$ off-axis from the sodium laser launch location, meaning that the imager can be used to provide a high resolution measurement of the sodium laser guide star profile. The dimensions of the elongated LGS when viewed using the wide-field imager is approximately $10 \times 500-1500$ pixels dependent on the depth of the sodium layer. The imager uses a PCO Edge 5.5 sCMOS camera that can run at frame rates up to $100 \mathrm{~Hz}$ full-frame, and faster with windowing.

\section{3 'Wendelstein' Laser Guide Star Unit}

The 'Wendelstein' Laser Guide Star Unit (WLGSU) ${ }^{9}$ is a sodium laser guide star launch system developed and operated by ESO that is installed $40 \mathrm{~m}$ from the WHT and emulates, in combination with CANARY, the LGS geometry that will be encountered at the ELT. This unique configuration allows tests of wave front sensing using highly-elongated laser guide stars, which is a characteristic issue encountered with large diameter telescopes. The WLGSU can also be operated independently of CANARY for LGS testing and development. The WLGSU also has two auxiliary $30 \mathrm{~cm}$ diameter telescopes that can be used to measure sodium LGS paramters (return flux, FWHM etc.) during operation. 


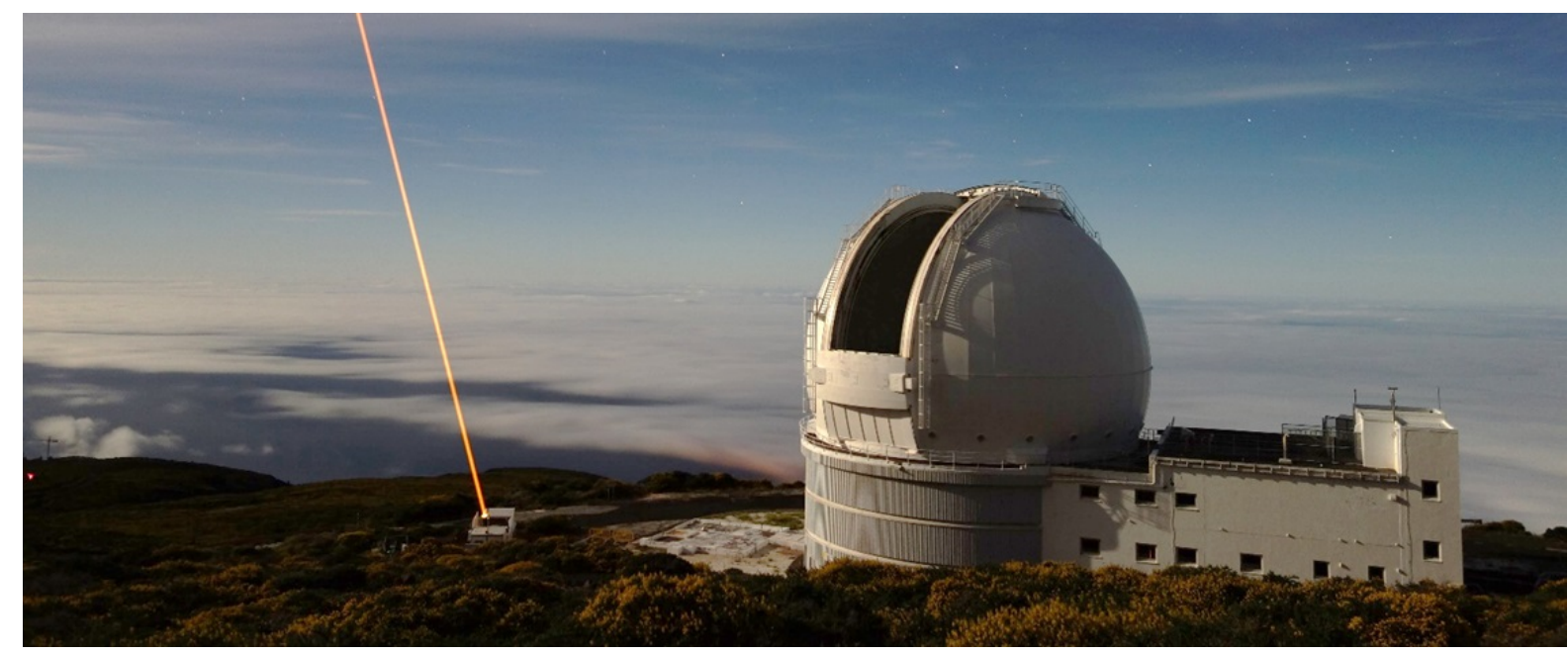

Figure 3. 'Wendelstein' Laser Guide Star Unit and William Herschel Telescope

\section{EXPERIMENTAL SUPPORT}

For each successful proposal awarded time on a facility, users will receive the following support:

- Pre-run: Successful applicants can expect to receive pre-run support of at least 2 staff months from the facility operator to assist with the design of their instrument e.g. providing details of opto-mechanical interfaces and assistance with software modification. Support staff will be experienced instrument developers who are familiar with the design and operation of the facility. Facility operators will coordinate the allocation of on-sky and setup time with the observatory.

- Commissioning and on-sky: Successful applicants will have staff on-site to assist with the installation of their instrument/experiment up to two weeks prior to the start of the on-sky run. Staff will be available to assist with the operation of the facility throughout this period. Costs for shipping user instruments to the facility (if applicable) are also provided.

- Post-run: Data recorded by the facility will be made available to users if needed. Assistance with the reduction and analysis of $\mathrm{AO}$ data in support of user publications will also be provided to ensure that instrument performance can be understood, and that users maximise the scientific output of the program.

The CANARY team will assist with operations and any modifications required to integrate their equipment/experiment with the facility, but their effort is limited so proposals that require significant amounts of effort from the CANARY team will be difficult to support. We advise you to discuss your proposals with them before submitting to see if what you propose is feasible within the time/effort constraints.

\section{APPLYING FOR TIME}

Users wishing to make use of these instrumentation facilities will apply to a time allocation committee that will rank proposals on the future scientific and technical impact of the proposed on-sky demonstration as well as their technical feasibility. Concepts must have reached a level of technical maturity where an on-sky demonstration would provide significant benefit to the future use of the technology, such as underpinning a future instrument proposal, or addressing issues that are difficult to recreate through simulation or in a laboratory environment.

The process is open to all European Union astronomers, and to non-EU astronomers if certain conditions are met, provided that they do not have automatic access to the facilities via national mechanisms. The committee may award time up to 2 years in advance to allow time for visitor instrumentation to be developed and tested before integration with the facility. Throughout the course of the ORP access program, both the William Herschel 
and Isaac Newton telescopes will have major new instruments commissioned that will utilise the majority of their observing time. The WHT will be available at times throughout each year when the WEAVE instrument is not installed. Access to the INT will be very limited once HARPS-3 has been installed, and any proposals requiring access to the INT should plan to go on-sky before the end of 2021. Specific on-sky dates cannot be requested and will be allocated by the observatory via their standard scheduling processes.

We are funded to offer two runs of 5 nights duration on the facilities installed on the WHT and INT telescopes. We may be able to support multiple visitor experiments on each facility simultaneously dependent on the requirements of each experiments and the expected level of support from the CANARY team required for installation and interfacing. The time allocation panel will assess if this is feasible before any time is awarded. We expect offer the first on-sky run in 2021/22 and a second in 2022/23.

Successful applicants will also receive some funding to pay for travel, subsistence and instrument shipping costs (if applicable).

\section{CONCLUSION}

Continued access to on-sky facilities for instrument development has been funded through the H2020 OPTICONRADIONET Pilot program. Individuals, research groups and institutes from across the EU will be able to apply for telescope time and dedicated technical support to test new instrumentation on-sky using three facilities installed at the ORM in La Palma, Spain. This includes the CANARY instrument installed on the $4.2 \mathrm{~m}$ William Herschel Telescope, the 2.5m Isaac Newton Telescope, and a sodium laser guide star system. Successful applicants will receive support in the design and integration of their experiment with these facilities, as well as funding to assist with travel and shipping. We expect to offer a total of 20 nights of telescope time equally split between the WHT and INT to be offered in two runs before 2023.

Please contact the authors of this paper if you wish to be kept informed of the upcoming call for proposals, which is expected to be issued in the first half of 2021.

\section{ACKNOWLEDGMENTS}

The on-sky access program has received funding from the European Union's Horizon 2020 research and innovation programme under grant agreement No 730890. This material reflects only the authors views and the Commission is not liable for any use that may be made of the information contained therein. The William Herschel and Isaac Newton telescopes are operated on the island of La Palma by the Isaac Newton Group of Telescopes in the Spanish Observatorio del Roque de los Muchachos of the Instituto de Astrofísica de Canarias.

\section{REFERENCES}

[1] Morris, T., Gendron, E., Rousset, G., Calia, D. B., Bardou, L., Centrone, M., Osborn, J., Chemla, F., Buey, T., Staykov, L., Bharmal, N., Townson, M., and Cohen, M., "Canary: An on-sky instrument development test bench open to the community," AO4ELT 6 (2019).

[2] Sinquin, B., Prengère, L., Kulcsár, C., Raynaud, H.-F., Gendron, E., Osborn, J., Basden, A., Conan, J.-M., Bharmal, N., Bardou, L., Staykov, L., Morris, T., Buey, T., Chemla, F., and Cohen, M., "On-sky results for adaptive optics control with data-driven models on low-order modes," Monthly Notices of the Royal Astronomical Society 498, 3228-3240 (08 2020).

[3] Haffert, S. Y., Harris, R. J., Zanutta, A., Pike, F., Bianco, A., Redaelli, E. M. A., Benoît, A., MacLachlan, D., Ross, C., Gris-Sánchez, I., Trappen, M., Xu, Y., Blaicher, M., Maier, P., Riva, G., Sinquin, B., Kulcsár, C., Bharmal, N. A., Éric Gendron, Staykov, L., Morris, T. J., Barboza, S., Muench, N., Bardou, L. F., Prengere, L., Raynaud, H.-F., Hottinger, P., Anagnos, T., Osborn, J., Koos, C., Thompson, R. R., Birks, T. A., Snellen, I. A. G., Keller, C. U., Close, L. M., and Males, J. R., "Multi-core fibre-fed integral-field unit (MCIFU): overview and first-light," in [Adaptive Optics Systems VII], 11448, International Society for Optics and Photonics, SPIE (2020). 
[4] Nayak, A. S., Sharma, T. K., Labadie, L., Piacentini, S., Corrielli, G., Osellame, R., Osborn, J., Morris, T. J., Éric Gendron, Buey, J.-T. M., Chemla, F., Cohen, M., Bharmal, N. A., Bardou, L. F., Staykov, L., Pedretti, E., Dinkelaker, A. N., Madhav, K. V., and Roth, M. M., "First on-sky results with an interferometric discrete beam combiner (DBC) at the William Herschel Telescope," in [Optical and Infrared Interferometry and Imaging VII], 11446, International Society for Optics and Photonics, SPIE (2020).

[5] Dubost, N., Bharmal, N. A., Langlois, M., Loupias, M., Schott, C., Tallon, M., Staykov, L., Bardou, L. F., Osborn, J., Chemla, F., Cohen, M., Buey, T., Éric Gendron, and Morris, T. J., "First on-sky results of the CAWS wavefront sensor on the CANARY experiment," in [Adaptive Optics Systems VII], 11448, International Society for Optics and Photonics, SPIE (2020).

[6] Langlois, M., Loupias, M., Dubost, N., Bharmal, N. A., Schotte, C., Tallon, M., Thiebaut, E., Bardou, L. F., Buey, T., Chemla, F., Cohen, M., Éric Gendron, Morris, T. J., Osborn, J., Staykov, L., Sinquin, B., Kulcsar, C., Prengère, L., and Raynaud, H.-F., "Mach-Zehnder wavefront sensor for XAO: From laboratory tests to on sky measurements using the SCAO capability of CANARY at the William Hershel Telescope," in [Adaptive Optics Systems VII], 11448, International Society for Optics and Photonics, SPIE (2020).

[7] Gendron, E., Morris, T., Basden, A., Vidal, F., Atkinson, D., Bitenc, U., Buey, T., Chemla, F., Cohen, M., Dickson, C., Dipper, N., Feautrier, P., Gach, J.-L., Gratadour, D., Henry, D., Huet, J.-M., Morel, C., Morris, S., Myers, R., Osborn, J., Perret, D., Reeves, A., Rousset, G., Sevin, A., Stadler, E., Talbot, G., Todd, S., and Younger, E., "Final two-stage MOAO on-sky demonstration with CANARY," in [Adaptive Optics Systems V], Marchetti, E., Close, L. M., and Véran, J.-P., eds., 9909, 126 - 142, International Society for Optics and Photonics, SPIE (2016).

[8] Shepherd, H. W., Osborn, J., Wilson, R. W., Butterley, T., Avila, R., Dhillon, V. S., and Morris, T. J., "Stereo-SCIDAR: optical turbulence profiling with high sensitivity using a modified SCIDAR instrument," Monthly Notices of the Royal Astronomical Society 437, 3568-3577 (11 2013).

[9] Calia, D. B., Guidolin, I., Friedenauer, A., Hager, M., Karpov, V., Pfrommer, T., Holzlöhner, R., Lewis, S., Hackenberg, W., Lombardi, G., Centrone, M., and Pedichini, F., "The ESO transportable LGS Unit for measurements of the LGS photon return and other experiments," in [Modern Technologies in Space- and Ground-based Telescopes and Instrumentation II], Navarro, R., Cunningham, C. R., and Prieto, E., eds., 8450, 606 - 617, International Society for Optics and Photonics, SPIE (2012). 